Ecología

\title{
Composición y estructura de las comunidades de poliquetos (Annelida) asociadas a fondos blandos de la costa occidental de la península de Baja California, México
}

\section{Composition and structure of polychaete communities (Annelida) associated with soft bottom of the West Coast of Baja California Peninsula, Mexico}

\author{
Andrea Prado-Navarro ${ }^{a}$, Victoria Díaz-Castañeda ${ }^{b}$, Antonio Leija-Tristán ${ }^{a}$ \\ y Jesús Angel de León-González ${ }^{\text {a,* }}$ \\ ${ }^{a}$ Laboratorio de Biosistemática, Facultad de Ciencias Biológicas, Universidad Autónoma de Nuevo León, \\ Apartado Postal 5 “F”, 66451, San Nicolás de los Garza, Nuevo León, México \\ ${ }^{\mathrm{b}}$ Centro de Investigación Científica y de Educación Superior de Ensenada, Carr. Ensenada-Tijuana 3918, \\ Zona Playitas, 22860 Ensenada, Baja California, México
}

Recibido el 20 de septiembre de 2014; aceptado el 6 de octubre de 2015

Disponible en Internet el 26 de febrero de 2016

\begin{abstract}
Resumen
Los poliquetos son uno de los grupos más importantes en el bentos desde cualquier punto de vista. Con el presente estudio se tiene el propósito de caracterizar la riqueza específica de la fauna de poliquetos de la costa occidental de Baja California, desde la boca de bahía Magdalena hasta punta Colonet, a través de recolectas realizadas por el crucero oceanográfico SIMSUP VIII. Se comparó latitudinal y batimétricamente el número de familias y especies encontradas en el estudio; asimismo, se realizó un análisis ecológico utilizando los índices de diversidad alfa, diversidad de Shannon-Wiener, dominancia de Simpson y equitatividad de Pielou. Se revisó un total de 60 estaciones; 30 de estas fueron positivas, las cuales resultaron en 3,709 organismos agrupados en 11 órdenes, 27 familias, 62 géneros y 99 especies. La familia Spionidae presenta la mayor riqueza con 4 géneros y 12 especies (12.2\% de la diversidad global y $42.7 \%$ de la abundancia relativa) para un total de 1,583 ejemplares. Paraprionospio pinnata fue la especie más abundante, frecuente y dominante (24.2\% del total de ejemplares). Del total de muestras, el $53.8 \%$ pertenecen a la zona centro, $26.9 \%$ a la sur y $19.2 \%$ a la norte. Se encontró la mayor riqueza (85.9\%) y abundancia (62.8\%) en la zona centro.

Derechos Reservados (C) 2015 Universidad Nacional Autónoma de México, Instituto de Biología. Este es un artículo de acceso abierto distribuido bajo los términos de la Licencia Creative Commons CC BY-NC-ND 4.0.
\end{abstract}

Palabras clave: Poliquetos; Diversidad; Pacífico mexicano

\section{Abstract}

Polychaetes are one of the most important groups of the benthos from any point of view. The present study aims to characterize the species richness of polychaete fauna of the West Coast of Baja California, from the mouth of Magdalena Bay to Punta Colonet. Collections were made by the Oceanographic Cruise SIMSUP VIII. We compared the number of families and species collected both bathymetricaly and latitudinally, we also performed a diversity analysis using several indexes such as alpha diversity, Pielou evenness, Simpson dominance and specific dominance. A total of 60 stations were revised, 30 of these were positive, finding 3,709 specimens grouped in 11 orders, 27 families, 62 genera and 99 species. Spionidae has the highest richness with 4 genera and 12 species $(12.2 \%$ of global diversity and $42.7 \%$ of the relative abundance) for a total of 1,583 specimens. Paraprionospio pinnata was the most abundant, frequent and dominant species ( $24.2 \%$ of all specimens). Of the total sample, $53.8 \%$ belong to the central zone, $26.9 \%$ to the southern one and $19.2 \%$ to the northern one. The highest richness ( $85.9 \%)$ and abundance (62.8\%) correspond to the central zone.

All Rights Reserved (C) 2015 Universidad Nacional Autónoma de México, Instituto de Biología. This is an open access item distributed under the Creative Commons CC License BY-NC-ND 4.0.

Keywords: Polychaetes; Diversity; Mexican Pacific

\footnotetext{
* Autor para correspondencia.

Correo electrónico: jesus.deleongn @uanl.edu.mx (J.A. de León-González).

La revisión por pares es responsabilidad de la Universidad Nacional Autónoma de México.
} 


\section{Introducción}

Los fondos blandos son considerados el hábitat bentónico más extenso sobre la plataforma continental, pendientes y cuencas marinas. Estos se encuentran constituidos por sedimentos arenosos y fangosos, los cuales difieren en el tamaño de grano, proporcionando así hábitats distintos para diferentes especies de organismos (Schiff, Allen, Zeng y Bay, 2000). El tipo de sedimento, incluyendo el tamaño de grano, porosidad, contenido orgánico y productos orgánicos que estos pudieron haber absorbido, se encuentra estrechamente relacionado con la distribución de los organismos que constituyen las comunidades. Los anélidos poliquetos son considerados un grupo dominante en dichas comunidades debido a su diversidad, abundancia y funcionalidad ecológica, lo que les permite una alta adaptabilidad a los diferentes hábitats (Díaz-Castañeda y Harris, 2004). Asimismo, los poliquetos forman parte de los grupos pioneros en las áreas en donde existe alguna perturbación ambiental de cualquier tipo (Díaz-Castañeda y Almeda-Jauregui, 1999; Heilskov y Holmer, 2001). Estos organismos son una parte esencial en las cadenas alimenticias, sirviendo, además, como importantes indicadores de las condiciones del medio ambiente (Díaz-Castañeda, de León y Solana, 2005) e intervienen directamente en el proceso de sedimentación, proporcionando un enlace entre la meiofauna y la macrofauna, permitiendo así el buen funcionamiento del medio (Cacabelos, Moreira y Troncoso, 2006). Considerando la importancia de los poliquetos en la estructura de los ecosistemas acuáticos, se han realizado diversos estudios en la plataforma continental de la costa occidental de la península de Baja California (De León-González, 1988, 1990, 1992, 1994a,b, 1998; Fauchald, 1968, 1970, 1972, 1982a,b; Hartman, 1939a,b, 1940, 1941, 1944a,b,c, 1950, 1956, 1957 y Hartman, 1961; Salazar-Vallejo, 1987; asimismo de De LeónGonzález, Hernández-Guevara y Rodríguez-Valencia, 2006; De León-González y Díaz-Castañeda, 1998; De León-González y Rodríguez-Valencia, 1996; De León-González y Solís-Weiss, 1998, 2000, 2001; Díaz-Castañeda, de León-González y SolanaArellano, 2014; Díaz-Castañeda y de León-González, 2007; Díaz-Castañeda y Harris, 2004; Díaz-Castañeda y San Martín, 2001; Díaz-Castañeda et al., 2005). En el presente estudio se determina la composición taxonómica de la fauna de poliquetos, los cambios espaciales a lo largo de la plataforma continental de la zona de estudio, así como las variaciones espaciales de la densidad y las afinidades faunísticas entre las localidades de muestreo, evaluando la diversidad alfa y determinando sus variaciones geográficas.

\section{Materiales y métodos}

En octubre de 1995, dentro del marco del proyecto SIMSUP VIII «Sistemas Marinos de Surgencias y su relación con Pelágicos menores» realizado en la costa occidental de la península de Baja California a bordo del buque oceanográfico «El Puma», fueron recolectadas muestras de fondos blandos en 60 estaciones por medio de una draga Smith-McIntyre $\left(0.1 \mathrm{~m}^{2}\right)$. Las estaciones se dividieron en zona norte: desde punta Colonet hasta
Tabla 1

Estaciones y posición geográfica establecidas dentro del margen del muestreo.

\begin{tabular}{|c|c|c|c|}
\hline Zona & Estación & Latitud N & Longitud $\mathrm{O}$ \\
\hline & 2 & $23^{\circ} 57^{\prime} 00.00^{\prime \prime}$ & $111^{\circ} 49^{\prime} 00.01^{\prime \prime}$ \\
\hline & 3 & $24^{\circ} 10^{\prime} 00.01^{\prime \prime}$ & $111^{\circ} 40^{\prime} 00.01^{\prime \prime}$ \\
\hline & 4 & $24^{\circ} 16^{\prime} 59.99^{\prime \prime}$ & $111^{\circ} 54^{\prime} 00.00^{\prime}$ \\
\hline \multirow[t]{12}{*}{ Sur } & 7 & $24^{\circ} 25^{\prime} 59.99^{\prime \prime}$ & $112^{\circ} 06^{\prime} 18.00^{\prime}$ \\
\hline & 8 & $24^{\circ} 15^{\prime} 00.00^{\prime \prime}$ & $112^{\circ} 13^{\prime} 30.00^{\prime \prime}$ \\
\hline & 9 & $24^{\circ} 04^{\prime} 00.01^{\prime \prime}$ & $112^{\circ} 22^{\prime} 00.01^{\prime \prime}$ \\
\hline & 10 & $24^{\circ} 10^{\prime} 59.99^{\prime \prime}$ & $112^{\circ} 33^{\prime} 18.00^{\prime}$ \\
\hline & 15 & $24^{\circ} 48^{\prime} 00.00^{\prime \prime}$ & $112^{\circ} 25^{\prime} 00.01^{\prime \prime}$ \\
\hline & 16 & $26^{\circ} 16^{\prime} 00.01^{\prime \prime}$ & $114^{\circ} 05^{\prime} 17.99^{\prime \prime}$ \\
\hline & 17 & $26^{\circ} 28^{\prime} 18.01^{\prime \prime}$ & $113^{\circ} 55^{\prime} 00.01^{\prime \prime}$ \\
\hline & 21 & $26^{\circ} 52^{\prime} 00.01^{\prime \prime}$ & $113^{\circ} 55^{\prime} 00.01^{\prime \prime}$ \\
\hline & 22 & $26^{\circ} 38^{\prime} 17.99^{\prime \prime}$ & $114^{\circ} 02^{\prime} 17.99^{\prime \prime}$ \\
\hline & 23 & $26^{\circ} 40^{\prime} 59.99^{\prime \prime}$ & $114^{\circ} 25^{\prime} 00.01^{\prime \prime}$ \\
\hline & 24 & $26^{\circ} 51^{\prime} 00.00^{\prime \prime}$ & $114^{\circ} 28^{\prime} 59.99^{\prime}$ \\
\hline & 27 & $27^{\circ} 01^{\prime} 00.01^{\prime \prime}$ & $114^{\circ} 43^{\prime} 00.01^{\prime \prime}$ \\
\hline \multirow[t]{12}{*}{ Centro } & 29 & $27^{\circ} 27^{\prime} 00.00^{\prime \prime}$ & $114^{\circ} 43^{\prime} 00.01^{\prime \prime}$ \\
\hline & 31 & $27^{\circ} 00^{\prime} 00.00^{\prime \prime}$ & $115^{\circ} 07^{\prime} 00.01^{\prime \prime}$ \\
\hline & 32 & $27^{\circ} 12^{\prime} 00.00^{\prime \prime}$ & $115^{\circ} 17^{\prime} 26.99^{\prime \prime}$ \\
\hline & 33 & $27^{\circ} 34^{\prime} 41.28^{\prime \prime}$ & $115^{\circ} 43^{\prime} 33.39^{\prime}$ \\
\hline & 35 & $27^{\circ} 49^{\prime} 18.01^{\prime \prime}$ & $115^{\circ} 09^{\prime} 00.00^{\prime \prime}$ \\
\hline & 36 & $27^{\circ} 37^{\prime} 00.01^{\prime \prime}$ & $115^{\circ} 19^{\prime} 59.99^{\prime \prime}$ \\
\hline & 37 & $27^{\circ} 49^{\prime} 18.01^{\prime \prime}$ & $115^{\circ} 30^{\prime} 00.00^{\prime \prime}$ \\
\hline & 38 & $28^{\circ} 00^{\prime} 00.00^{\prime \prime}$ & $115^{\circ} 19^{\prime} 59.99^{\prime \prime}$ \\
\hline & 39 & $28^{\circ} 00^{\prime} 00.00^{\prime \prime}$ & $115^{\circ} 03^{\prime} 00.00^{\prime}$ \\
\hline & 44 & $29^{\circ} 46^{\prime} 00.01^{\prime \prime}$ & $115^{\circ} 12^{\prime} 01.80^{\prime \prime}$ \\
\hline & 50 & $29^{\circ} 55^{\prime} 00.01^{\prime \prime}$ & $116^{\circ} 10^{\prime} 59.99^{\prime \prime}$ \\
\hline & 52 & $30^{\circ} 19^{\prime} 59.99^{\prime \prime}$ & $116^{\circ} 06^{\prime} 00.00^{\prime \prime}$ \\
\hline \multirow[t]{3}{*}{ Norte } & 55 & $30^{\circ} 32^{\prime} 17.99^{\prime \prime}$ & $116^{\circ} 10^{\prime} 59.99^{\prime \prime}$ \\
\hline & 56 & $30^{\circ} 46^{\prime} 59.99^{\prime \prime}$ & $116^{\circ} 16^{\prime} 18.01^{\prime \prime}$ \\
\hline & 60 & $31^{\circ} 16^{\prime} 00.01^{\prime \prime}$ & $116^{\circ} 31^{\prime} 00.01^{\prime \prime}$ \\
\hline
\end{tabular}

punta San Antonio; zona centro: desde punta Eugenia hasta San Hipólito, y zona sur: en la boca de bahía Magdalena (fig. 1). En la tabla 1 se muestran las coordenadas de las 30 estaciones positivas referentes en este trabajo. Cada transecto se dividió batimétricamente en 4 intervalos de profundidad: de 0-49 m, de 50-99 m, de 100-149 m y de 150-200 m, tal como ha sido sugerido por Hernández-Alcántara (2002); McConnaughey (1978) y Ross (1977). La cuantificación de organismos recolectados se llevó a cabo por especie y su registro en las tablas respectivas se presenta en términos de densidad (orgs $/ 0.1 \mathrm{~m}^{2}$ ), que es el área de muestreo de la draga Smith-McIntyre utilizada en la toma de muestras. La representación y análisis de la distribución espacial de la densidad y el número de especies se realizó por medio de mapas de distribución. Para esto, se incluyeron diversos índices y tratamientos estadísticos tales como: diversidad alfa, equitatividad de Pielou y dominancia de Simpson. Con la finalidad de analizar las variaciones norte-sur de la fauna de poliquetos y comparar las diferencias en la composición de especies a lo largo del margen peninsular, se evaluó la diversidad Beta (Gray, 2000). La utilización de la diversidad Beta en la costa occidental de la península de Baja California permitió detectar cambios bióticos en sentido norte-sur, por medio del índice propuesto por Wilson y Shmida (1984). Para seleccionar adecuadamente las especies representativas de una comunidad se determinaron las especies dominantes tanto en las localidades de muestreo como 


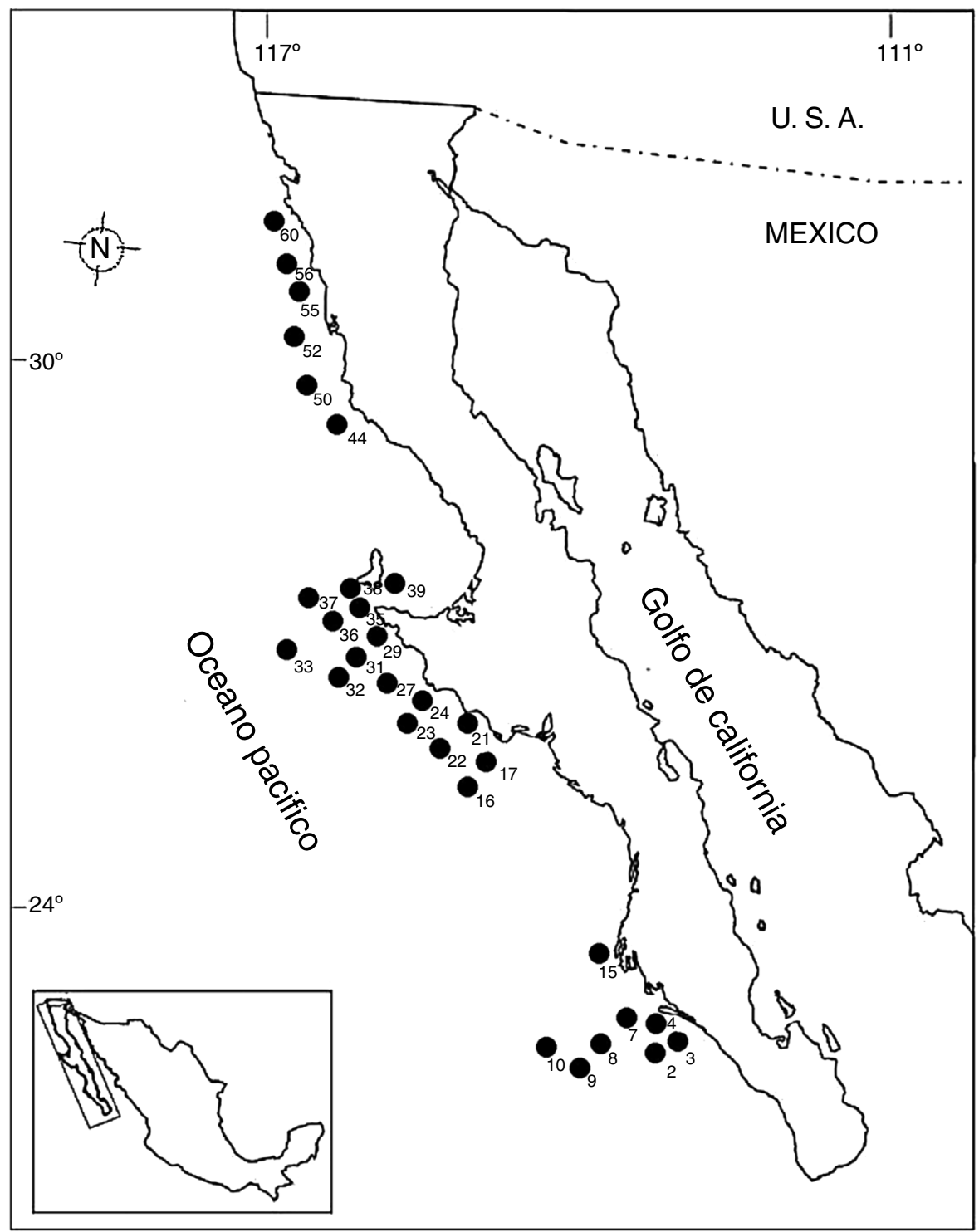

Figura 1. Área de estudio (punta Colonet a boca de bahía Magdalena) en el que se distinguen las 3 zonas de muestreo.

en las diferentes regiones geográficas mediante la dominancia específica.

\section{Resultados}

Análisis global de la riqueza y abundancia específica

Se examinaron un total de 3,709 organismos incluidos en 62 géneros y 99 especies (Anexo 1). La familia Spionidae presentó la mayor riqueza, constituida por 12 especies y 4 géneros: Minuspio cirrifera, Minuspio perkinsi, Paraprionospio alata, Paraprionospio pinnata, Prionospio cristata, Prionospio dubia, Prionospio jubata, Prionospio malmgreni, Prionospio vermillionensis, Spiophanes bombyx, Spiophanes kroeyeri y Spiophanes wigleyi, las mismas que componen el $12.2 \%$ de la diversidad global y el $42.7 \%$ de la abundancia relativa (1,583 organismos). Las familias Syllidae (Exogone dispar, Opisthosyllis brunnea, Opisthosyllis japonica, Syllis caeca, Syllis gracilis, Trypanosyllis aeolis y Trypanosyllis zebra), Ampharetidae (Amphicteis scaphobranchiata, Eclysippe trilobata, Lysippe labiata, Melinna oculata, Melinna tentaculata, Melinnampharete gracilis y Paralysippe annectens), Cirratulidae (Aphelochaeta monilaris, Aphelochaeta parvus, Caulleriella gracilis, Chaetozone corona, Chaetozone setosa, Monticellina serratiseta y Monticellina tesselata) y Maldanidae (Clymenella complanata, Clymenella mucosa, Clymenura gracilis, Maldane sarsi, Praxillella gracilis, Praxillella pacifica y Rhodine bitorquata) agrupan el $28.2 \%$ del total de especies y $24.0 \%$ de abundancia - 889 organismos - (fig. 2). Las familias que mostraron la menor diversidad específica fueron Capitellidae (Capitella sp.), Chaetopteridae (Spiochaetopterus costarum), Flabelligeridae (Pherusa neopapillata), Scalibregmatidae (Scalibregma inflatum), Serpulidae (Hydroides gracilis), Sternapsidae (Sternaspis fossor), todas con una sola especie, englobando el $7.7 \%$ (287) de abundancia del total de individuos. 


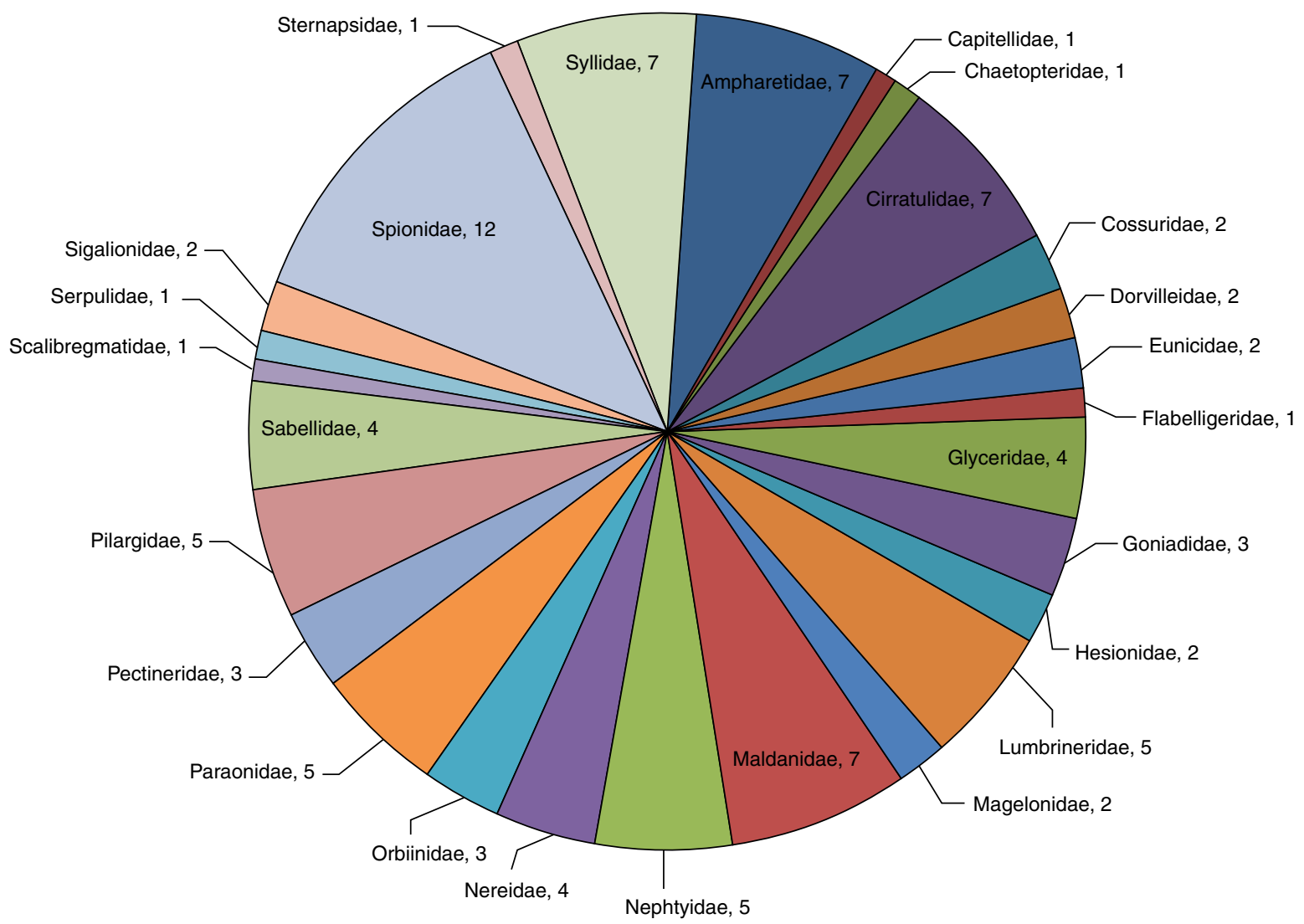

\begin{tabular}{|c|c|c|c|c|c|}
\hline$\square$ Ampharetidae & $\square$ Dorvilleidae & $\square$ Hesionidae & $\square$ Nereidae & $\square$ Sabellidae & $\square$ Sternapsidae \\
\hline$\square$ Capitellidae & $\square$ Eunicidae & $\square$ Lumbrineridae & $\square$ Orbiinidae & $\square$ Scalibregmatidae & $\square$ Syllidae \\
\hline$\square$ Chaetopteridae & $\square$ Flabelligeridae & $\square$ Magelonidae & $\square$ Paraonidae & $\square$ Serpulidae & \\
\hline$\square$ Cirratulidae & $\square$ Glyceridae & $\square$ Maldanidae & $\square$ Pectineridae & $\square$ Sigalionidae & \\
\hline$\square$ Cossuridae & $\square$ Goniadidae & $\square$ Nephtyidae & $\square$ Pilargidae & $\square$ Spionidae & \\
\hline
\end{tabular}

Figura 2. Análisis global de la riqueza y abundancia específica.

Análisis de la riqueza específica en la zona sur

De las muestras obtenidas en esta zona se identificaron 788 organismos $(21.2 \%)$, representando 26 familias y 61 especies $(61.6 \%)$, donde 7 de estas corresponden a Spionidae (M. cirrifera, $M$. perkinsi, $P$. alata, $P$. pinnata, $P$. malmgreni, $S$. bombyx y $S$. kroeyeri), constituyendo el $11.5 \%$ de la diversidad específica y el $35.9 \%$ de abundancia relativa - 283 organismos - de la zona, mientras que Syllidae $(E$. dispar, S. caeca, S. gracilis y T. aeolis), Ampharetidae (E. trilobata, M. tentaculata, M. gracilis y P. annectens), Lumbrineridae (Lumbrinereis crassidentata, Lumbrinereis erecta, Lumbrinereis latreilli y Ninoe longibranchiata) y Sabellidae (Euchone incolor, Megalomma pigmentum, Paradialychone ecaudata y Pseudopotamilla reniformis) equivalen al $26.2 \%$ de la riqueza en la zona y $17.9 \%$ de abundancia relativa -141 organismosAsimismo, Cirratulidae (A. parvus, C. setosa y M. tesselata), Goniadidae (Glycinde polygnatha, Goniada brunnea y Goniada maculata), Maldanidae (M. sarsi, P. gracilis y P. pacifica), Orbiinidae (Leitoscoloplos kerguelensis, Leitoscoloplos pugettensis y Scoloplos treadwelli) y Pilargidae (Ancistrosyllis jonesi, Sigambra bassi y Sigambra tentaculata) constituyen el $24.6 \%$ en el número de especies y $22.7 \%$ de abundancia relativa -179 organismos- Sternapsidae (S. fossor), Capitellidae (Capitella sp.), Chaetopteridae (S. costarum), Dorvilleidae (Schistomeringos annulata), Eunicidae (Eunice vittata), Flabelligareidae (P. neopapillata), Hesionidae (Hesione intertexta), Magelonidae (Magelona pacifica), Pectineridae (Amphictene auricoma), Scalibregmatidae (S. inflatum) y Serpulidae $(H$. gracilis) corresponden al $17.9 \%$ de la riqueza específica y $13.1 \%$ del número de organismos - 103 organismos- (fig. 3).

\section{Análisis de la riqueza específica en la zona norte}

En la zona norte se identificaron un total de 592 organismos (16\%) pertenecientes a 23 familias y 60 especies, donde la familia Spionidae representa el $18.3 \%$ de la riqueza específica de la zona (M. cirrifera, P. alata, P. pinnata, $P$. cristata, P. dubia, $P$. jubata, P. malmgreni, P. vermillionensis, $S$. bombyx, S. kroeyeri y $S$. wigleyi). Ampharetidae (A. scaphobranchiata, E. trilobata, L. labiata, M. oculata, $M$. gracilis y $P$. annectens) y Maldanidae (C. complanata, C. mucosa, C. gracilis, M. sarsi, $P$. 


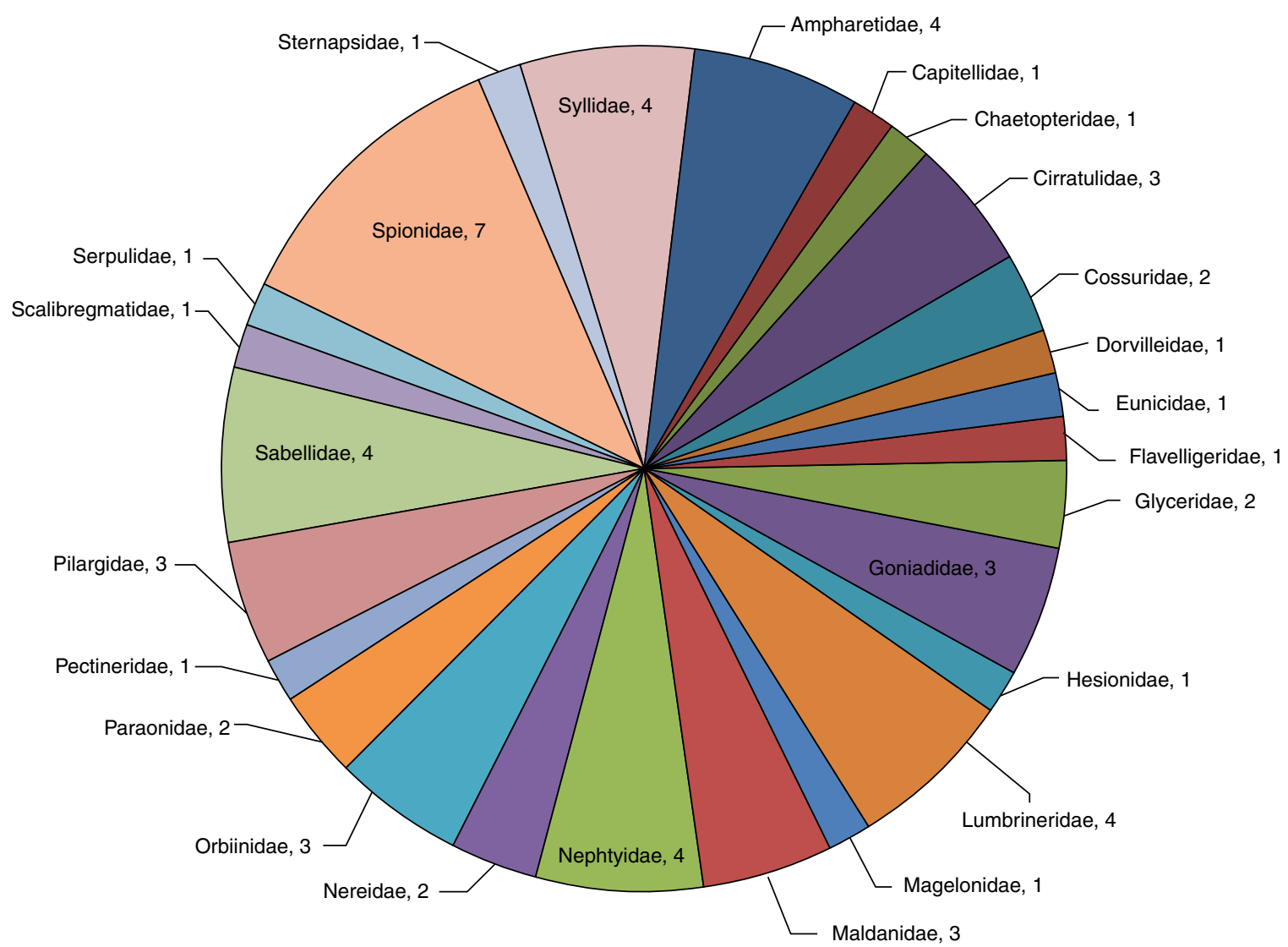

Figura 3. Análisis de la riqueza y abundancia para la zona sur.

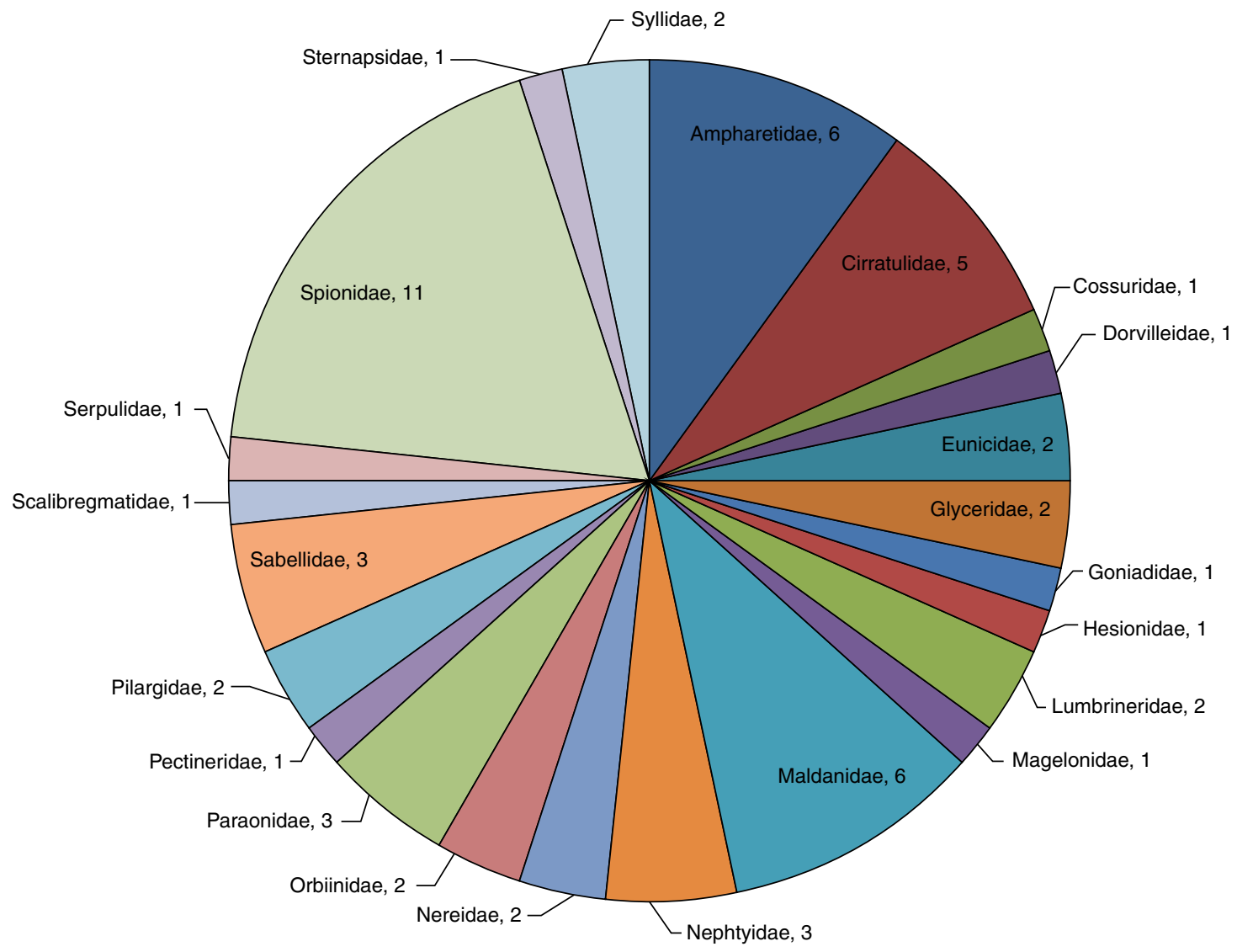

Figura 4. Análisis de la riqueza y abundancia para la zona norte. 


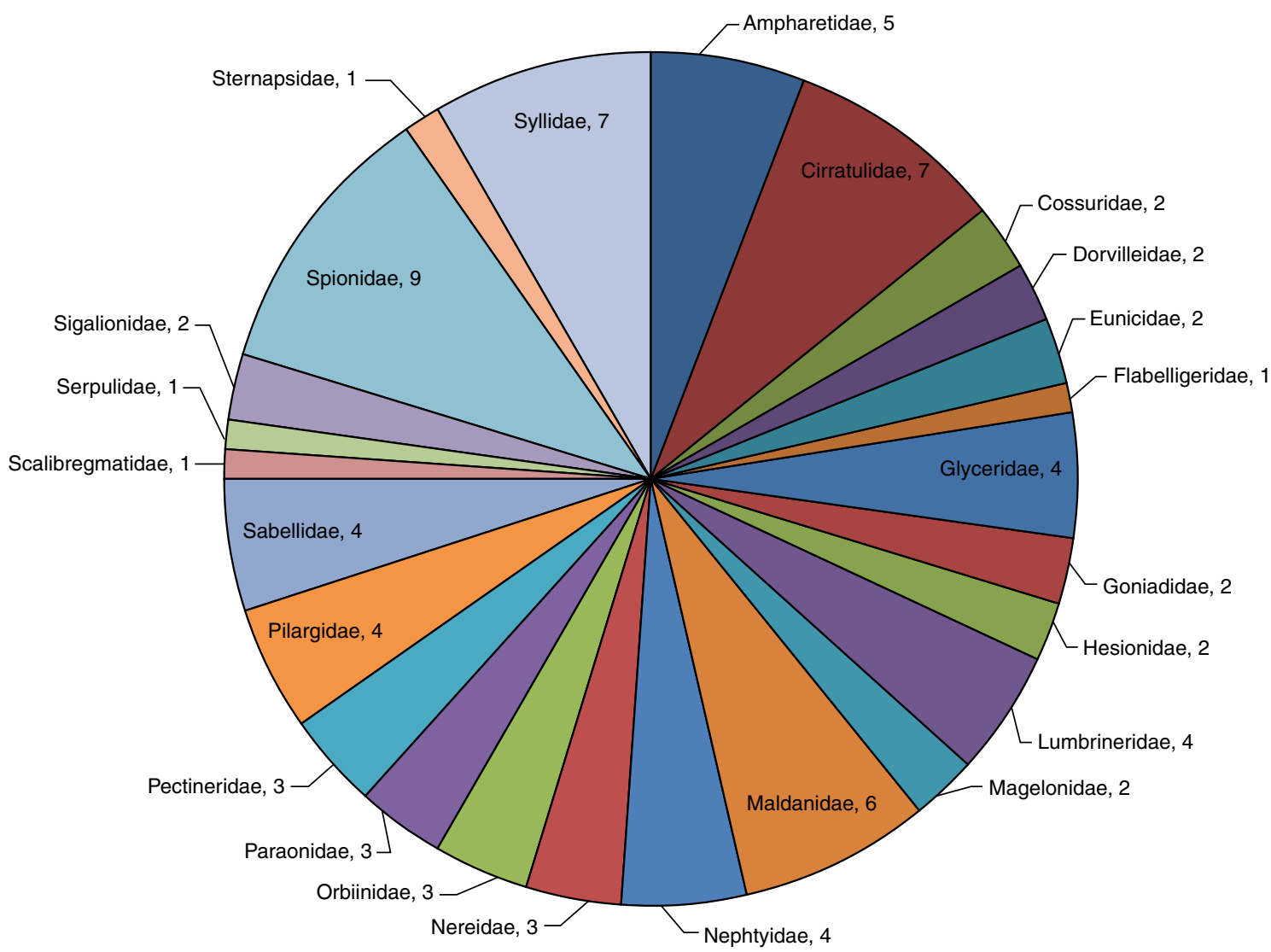

Figura 5. Análisis de la riqueza y abundancia para la zona centro.

pacifica y $R$. bitorquata), concentran el $20.0 \%$ de la riqueza y el $11.3 \%$ de la abundancia -67 organismos-. La familia Cirratulidae engloba 5 especies (A. monilaris, A. parvus, $C$. gracilis, $C$. setosa y $M$. tesselata), significando el $8.3 \%$ de la riqueza y el $10.8 \%$ de la abundancia - 64 organismosSternapsidae (S. fossor), Dorvilleidae ( $S$. annulata), Hesionidae (Podarkeopsis glabra), Magelonidae (Magelona suculata), Pectineridae (Pectinaria californiensis), Scalibregmatidae ( $S$. inflatum), Cossuridae (Cossura brunnea), Goniadidae (G. maculata) y Serpulidae ( $H$. gracilis) resultaron con una sola especie presente en esta zona, lo que representa el $14.94 \%$ de la riqueza y el $17.1 \%$ de la abundancia - 101 organismos- (fig. 4).

\section{Análisis de la riqueza específica en la zona centro}

De esta zona se identificaron un total de 2,329 organismos (62.8\%) pertenecientes a 25 familias y 82 especies (82.8\%). La familia Spionidae, igual que en las otras zonas, fue la mejor representada con 9 especies (M. cirrifera, $M$. perkinsi, $P$. alata, $P$. pinnata, $P$. malmgreni, $P$. vermillionensis, $S$. bombyx, $S$. kroeyeri y $S$. wigleyi) equivalentes al $11.0 \%$ de la riqueza total de la zona y el $46.8 \%$ de la abundancia - 1,089 organismos- Syllidae $(E$. dispar, O. brunnea, O. japonica, S. caeca, S. gracilis, T. aeolis y T. zebra) y Cirratulidae (A. monilaris, A. parvus, C. gracilis, $C$. corona, $C$. setosa, $M$. serratiseta y $M$. tesselata) están constituidas por 7 especies cada una, ambas equivalentes al $17.1 \%$ de la riqueza y $15.2 \%$ de la abundancia -354 organismos-. Maldanidae con 6 especies (C. complanata, C. mucosa, C. gracilis,
M. sarsi, $P$. pacifica y $R$. bitorquata), lo que representa $7.3 \%$ de la riqueza y $7.5 \%$ de la abundancia -174 organismos- L Las familias Sternapsidae (S. fossor), Flabelligeridae (P. neopapillata), Scalibregmatidae (S. inflatum) y Serpulidae (H. gracilis) resultaron con una sola especie presente en esta zona, lo que representa $4.9 \%$ del total de la riqueza y $9.1 \%$ de la abundancia -212 organismos- (fig. 5).

\section{Estimación global de índices con importancia biológica específica}

Los resultados indican que la familia Maldanidae presentó la mayor diversidad de Shannon-Wiener con un valor de 1.76, mismo que contrasta con la obtención de un dato bajo de dominancia (0.194) y corroborado por un alto valor de equitatividad correspondiente a 0.9032 . Otras familias donde también se observan datos sobresalientes de diversidad y equitatividad son Cirratulidae, Spionidae, Nephtyidae y Sabellidae. De la misma forma, las familias con valores bajos de diversidad y equitatividad, así como una alta dominancia son Dorvilleidae y Eunicidae, tal como se observa en la (fig. 6).

\section{Estimación de índices con importancia biológica en la zona} sur

En la zona sur se recolectaron un total de 26 familias, de las cuales Spionidae resultó la más diversa, con un total de 7 

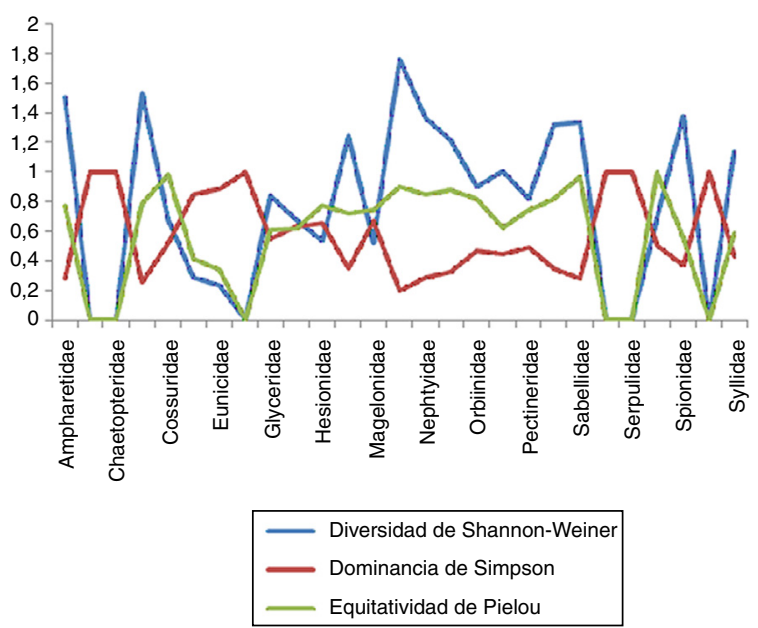

Figura 6. Estimación de índices con importancia biológica a nivel de especie a lo largo del estudio.

especies, y presentó un valor de 1.652, lo que nos indica una equitatividad elevada (0.849) en las 7 estaciones en las que estuvo presente y una dominancia menor (0.214). En el caso de la familia Sabellidae comprendió un total de 4 especies en 4 estaciones, evidenciando que es la segunda familia con valores de diversidad y equitatividad mayores (1.316 y 0.949 , respectivamente) y una dominancia menor (0.286). Dentro de las familias que presentaron la menor diversidad se encuentran Nereididae con 2 especies en 3 estaciones, con un valor de 0.673 y una equitatividad igualmente baja ( 0.52 ); sin embargo, esto nos muestra que existe una alta dominancia entre las especies presentes (0.917). En el caso de Capitellidae, Chaetopteridae, Dorvilleidae, Eunicidae, Hesionidae, Magelonidae, Pectineridae, Scalibregmatidae, Serpulidae y Sternapsidae se obtuvieron niveles tanto de diversidad como de equitatividad iguales a $0 \mathrm{y}$ una dominancia igual a 1 , lo que nos indica que solo se cuenta con una sola especie para cada caso (fig. 7).

\section{Estimación de índices con importancia biológica en la zona centro}

En la zona centro se encontraron un total de 25 familias, de las cuales Cirratulidae resultó la más diversa con un valor de 1.537 $\mathrm{y}$ un total de 7 especies en 9 estaciones, lo que nos indica una equitatividad elevada (0.79) y una dominancia menor (0.266). En el caso de Maldanidae presentó un total de 6 especies en 8 estaciones para esta zona, mostrando que es la segunda familia con valores de diversidad y equitatividad mayores (1.421 y 0.793 , respectivamente) y una dominancia menor (0.284). Dentro de las familias que presentaron la menor diversidad se encuentran Eunicidae con 2 especies en 5 estaciones, con un valor de 1.732 y una equitatividad igualmente baja (0.249); sin embargo, esto nos muestra que existe una alta dominancia entre las especies presentes (0.9201), ya que una de sus 2 especies presenta una mayor cantidad de organismos en esta zona (fig. 8). En el caso de Flabelligeridae, Scalibregmatidae, Serpulidae y Sternapsidae se obtuvieron niveles tanto de diversidad como de equitatividad iguales a $0 \mathrm{y}$ una dominancia igual a 1 , lo que nos
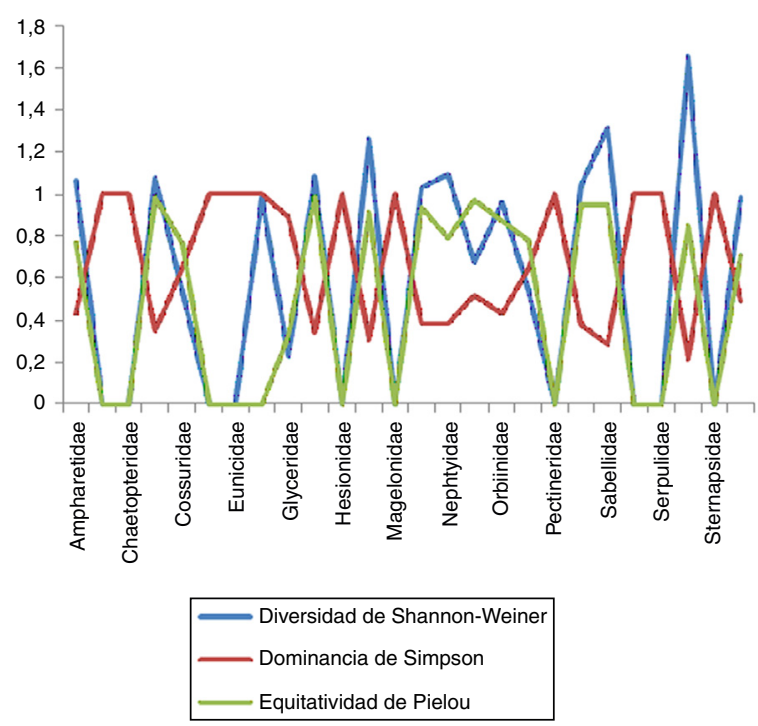

Figura 7. Estimación de índices con importancia biológica a nivel de especie a lo largo de la zona sur.

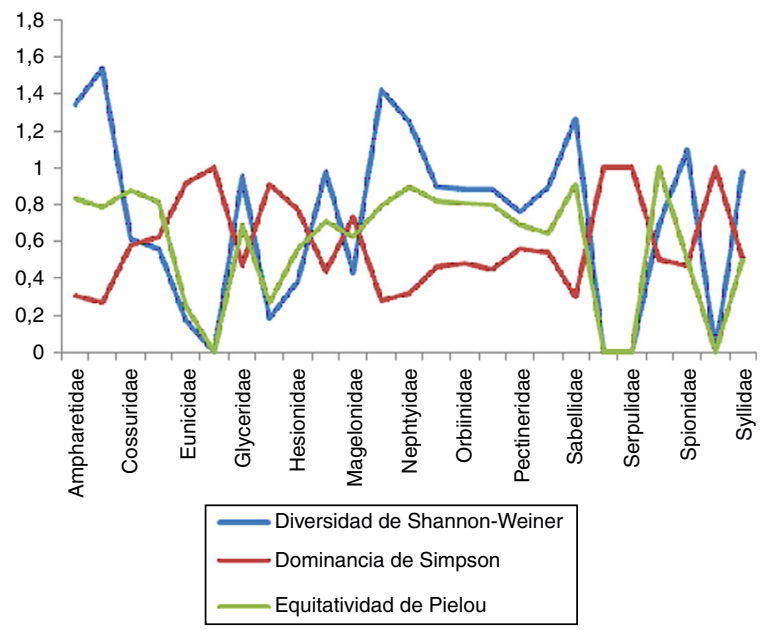

Figura 8. Estimación de índices con importancia biológica a nivel de especie a lo largo de la zona centro.

indica que solo se cuenta con una sola especie de organismo para cada caso.

\section{Estimación de índices con importancia biológica en la zona norte}

En la zona norte se encontraron un total de 23 familias, de las cuales Maldanidae resultó la más diversa con un valor de 1.589 y un total de 6 especies en 4 estaciones, lo que nos indica una equitatividad elevada (0.886) y una dominancia menor $(0.218)$. En el caso de Spionidae, arrojó un total de 11 especies en 5 estaciones para esta zona, mostrando que es la segunda familia con valores de diversidad y equitatividad mayores (1.466 y 0.611 , respectivamente) y una dominancia menor (0.341), debido a que 3 de estas especies presentan una mayor abundancia con respecto al resto. Dentro de las familias que presentaron la menor diversidad se encuentran Eunicidae con 2 especies en 2 estaciones, con un valor de 0.500 y una equitatividad alta (0.721); sin embargo, 


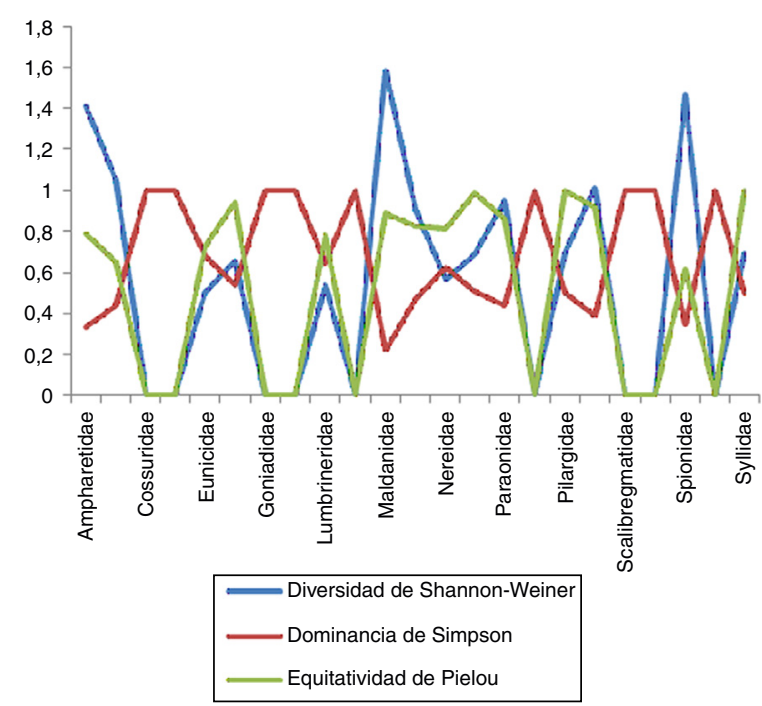

Figura 9. Estimación de índices con importancia biológica a nivel de especie a lo largo de la zona norte.

esto nos muestra que existe una alta dominancia entre las especies presentes (0.680). En el caso de Cossuridae, Dorvilleidae, Goniadidae, Hesionidae, Magelonidae, Pectineridae, Scalibregmatidae, Serpulidae y Sternapsidae se obtuvieron niveles tanto de diversidad como de equitatividad iguales a 0 y una dominancia igual a 1 , lo que nos indica que solo se cuenta con una sola especie de organismo para cada caso (fig. 9).

\section{Análisis batimétrico con base en la estimación de índices de importancia biológica}

De las 60 estaciones de muestreo durante el crucero SIMSUP VIII, en 30 se obtuvo por lo menos un ejemplar de poliqueto, donde el $53.3 \%$ corresponden a la zona centro, el $26.7 \%$ a la sur y el $20 \%$ a la norte. Asimismo, la mayor riqueza $(85.9 \%)$ y abundancia $(62.8 \%)$ fueron producto de recolectas realizadas en la zona centro. Cabe señalar que estas diferencias con respecto a las otras 2 zonas podrían atribuirse a que el 56\% de las estaciones muestreadas fueron en esta área. En el estudio realizado por Rodríguez-Valencia (1994), equivalente a la zona centro y comparado con el de Hernández-Guevara (1994), correspondiente a la zona norte, también fue superior en riqueza específica y abundancia, tal como sucedió en el presente estudio (tabla 2). Batimétricamente, el intervalo 50-99 m para el área total y para las 3 zonas presentó la mayor riqueza y abundancia específica (tabla 3), así como la mayor diversidad estimada con base en el índice Shannon-Wiener y Simpson.

\section{Discusión}

La costa occidental de Baja California hasta mediados de 1980 concentraba aproximadamente 236 especies de poliquetos (Salazar-Vallejo, de León-González y Salaices-Polanco, 1989). Barbosa-López (2008) llevó a cabo la revisión de las bases de datos de los registros realizados entre 1901 y 2004 en la plataforma continental de la costa occidental de Baja California, registrando un total de 491 especies, por lo que el listado elaborado en el presente estudio corresponde al 20.2\% de tales especies (Anexo 1). En estudios de ciclos cortos, RodríguezValencia (1994) identificó un total de 20 familias en el área correspondiente a bahía de San Juanico, en la costa occidental de Baja California Sur, de las cuales el $85 \%$ de ellas son las mismas a las encontradas en el presente trabajo. Asimismo, HernándezGuevara (1994) identificó 23 familias de poliquetos en la bahía Sebastián Vizcaíno, donde el 88\% también están presentes en esta investigación. Con el trabajo de De León-González (1994a) se obtuvo un traslape del $85 \%$ de las familias documentadas en ambos estudios.

El 64.5\% de las especies de poliquetos identificadas en el presente estudio pertenecen a 10 familias: Spionidae, Maldanidae, Ampharetidae, Syllidae, Cirratulidae, Nephtyidae, Paraonidae, Pilargiidae, Lumbrineridae y Nereididae, las cuales, en su mayoría, son dominantes en sustratos blandos. Rodríguez-Valencia (1994) obtiene una estimación del 62.2\% en las 10 familias con mayor riqueza, donde 7 de ellas aparecen en ambos listados, y Hernández-Guevara (1994) obtiene el $67.3 \%$ con 5 familias muestreadas en ambos trabajos. En la misma área de estudio, Barbosa-López (2008) registra que Spionidae, Paraonidae, Ampharetidae, Cirratulidae, Nereidae y Syllidae contribuyen con el 32.8\% de las especies. Hernández-Alcántara (2002), en el golfo de California, también señala dentro de las primeras 10 familias con la mayor riqueza y número de organismos a Spionidae, Lumbrineridae, Nereididae, Paraonidae y Cirratulidae, mismas que representan el $27.6 \%$ de las especies.

De las 27 familias, Capitellidae, Sternapsidae, Serpulidae, Flabelligeridae, Chaetopteridae y Scalibregmatidae estuvieron representadas por una sola especie; sin embargo, Sternapsidae presentó 198 organismos y el resto, menos de 20. Comparativamente, otros estudios llevados a cabo por Rodríguez-Valencia (1994) arrojaron un total de 55\% de similitud, lo que equivale a 11 familias con una sola especie. Hernández-Guevara (1994) mostró una similitud del $60 \%$, lo equivalente a 15 familias, las cuales solo presentaron una especie. De León-González (1994a) documenta para el área de punta Eugenia hasta la punta de Cabo Falso una similitud de $39.4 \%$ o lo equivalente a 13 familias. Hernández-Alcántara (2002) igualmente registra a Sternapsidae, Serpulidae y Chaetopteridae con una sola especie para el golfo de California.

Uno de los resultados preponderantes del análisis de la poliquetofauna fueron los datos sobresalientes de riqueza y abundancia que representa la familia Spionidae, debido a que constituye el $12.2 \%$ de las 99 especies identificadas y el $42.7 \%$ de los 3,709 organismos. Información similar fue documentada por De León-González (1994a), contabilizando 10.6 y 37.8\% de riqueza y abundancia, respectivamente. Igualmente, HernándezGuevara (1994) identificó 5 especies (10.9\%) de 171 organismos de esta familia (25.8\%). Asimismo, Rodríguez-Valencia (1994) examinó 712 ejemplares $(66.4 \%)$ pertenecientes a 4 especies. Cabe destacar que la mayor presencia de esta familia está fundamentada en la diversidad de hábitat que ocupa tanto latitudinal como batimétricamente en la plataforma continental, dominando ampliamente los fondos blandos de aguas someras y profundas, tal como es señalado por Hernández-Valdez (2008). 
Tabla 2

Análisis batimétrico con base en la estimación de índices de importancia biológica.

\begin{tabular}{|c|c|c|c|c|c|c|}
\hline Profundidad & Especies & $\begin{array}{l}\text { Número de } \\
\text { individuos }\end{array}$ & $\begin{array}{l}\text { Dominancia } \\
\text { de Simpson }\end{array}$ & $\begin{array}{l}\text { Diversidad de } \\
\text { Shannon-Weiner }\end{array}$ & $\begin{array}{l}\text { Diversidad de } \\
\text { Simpson }\end{array}$ & $\begin{array}{l}\text { Equitatividad } \\
\text { de Pielou }\end{array}$ \\
\hline $0-49$ & 68 & 386 & 0.03801 & 3.703 & 0.962 & 0.8777 \\
\hline $50-99$ & 92 & 2,690 & 0.05317 & 3.55 & 0.9468 & 0.785 \\
\hline $100-149$ & 2 & 3 & 0.5556 & 0.6365 & 0.4444 & 0.9183 \\
\hline $150-200$ & 34 & 630 & 0.4622 & 1.599 & 0.5378 & 0.4534 \\
\hline
\end{tabular}

Tabla 3

Análisis batimétrico por zona con base en la estimación de índices de importancia biológica.

\begin{tabular}{|c|c|c|c|c|c|c|c|}
\hline Zona & Profundidad & Especies & Número de individuos & $\begin{array}{l}\text { Dominancia } \\
\text { de Simpson }\end{array}$ & $\begin{array}{l}\text { Diversidad de } \\
\text { Shannon-Weiner }\end{array}$ & $\begin{array}{l}\text { Diversidad de } \\
\text { Simpson }\end{array}$ & $\begin{array}{l}\text { Equitatividad } \\
\text { de Pielou }\end{array}$ \\
\hline & $0-49$ & 37 & 177 & 0.06566 & 3.107 & 0.9343 & 0.8603 \\
\hline \multirow[t]{3}{*}{ Sur } & $50-99$ & 51 & 604 & 0.05127 & 3.352 & 0.9487 & 0.8524 \\
\hline & $100-149$ & 1 & 1 & 1 & 0 & 0 & 0 \\
\hline & $0-49$ & 42 & 107 & 0.04096 & 3.447 & 0.959 & 0.9222 \\
\hline \multirow[t]{3}{*}{ Centro } & $\begin{array}{c}50-99 \\
100-149\end{array}$ & 73 & 1,592 & 0.05494 & 3.407 & 0.9451 & 0.794 \\
\hline & $150-200$ & 34 & 630 & 0.4622 & 1.599 & 0.5378 & 0.4534 \\
\hline & $0-49$ & 29 & 102 & 0.09035 & 2.831 & 0.9097 & 0.8406 \\
\hline \multirow[t]{3}{*}{ Norte } & $50-99$ & 51 & 494 & 0.09086 & 3.027 & 0.9091 & 0.7699 \\
\hline & $100-149$ & 1 & 2 & 1 & 0 & 0 & 0 \\
\hline & $150-200$ & & & & & & \\
\hline
\end{tabular}

P. pinnata es la especie más abundante, frecuente y dominante entre las familias de poliquetos obtenidas en este estudio y representa el $24.2 \%$ del número total de organismos. En los estudios realizados por De León-González (1994a), Donath-Hernández y Loya-Salinas (1989), Encalada-Fleites y Millán-Núñez (1990) y Rodríguez-Valencia (1994), también se identificó a $P$. pinnata como la especie más abundante.

Del total de especies identificadas en la zona sur, la familia Spionidae fue la más diversa, así como en el trabajo de De León-González (1994a) que menciona a estos organismos sedimentívoros subsuperficiales como los más abundantes y con mayor riqueza de especies en la plataforma continental de la costa oeste de Baja California, sobre todo en sustratos blandos. La zona centro comprendió un total de 85 especies de Spionidae; nuevamente es la mejor representada, seguida por Cirratulidae. Eunicidae en esta área resultó la familia con una menor diversidad de especies. Rodríguez-Valencia (1994) menciona que esta zona presenta una acumulación de materia orgánica formando fondos blandos en su mayor parte, haciéndola homogénea y logrando el enriquecimiento del sustrato, favoreciendo así el desarrollo de un mayor número de especies con hábitos sedentarios, las cuales van desapareciendo en los estratos más profundos. En la zona norte se identificaron un total de 60 especies, de las cuales 11 pertenecen a la familia Spionidae; esta presenta la mayor diversidad al igual que en el estudio realizado por HernándezGuevara (1994) para el área de punta María a punta Eugenia, quien menciona que el indicador de la magnitud en que los sustratos son favorables a una especie o de la adaptación de la especie a ese sustrato es la densidad de organismos por unidad de muestreo.

\section{Agradecimientos}

El primer autor agradece al Conacyt el apoyo financiero por medio de una beca doctoral durante el desarrollo de este trabajo. Asimismo, Agradecemos al personal técnico del B/O El Puma por el apoyo en la obtención del material registrado en este trabajo. Agradecemos los comentarios y sugerencias de 2 revisores anónimos que mejoraron el manuscrito.

\section{Anexo 1. Lista de especies encontradas durante el estudio}

\begin{tabular}{c}
\hline $\begin{array}{c}\text { Phylum Annelida } \\
\text { Clase Polychaeta }\end{array}$ \\
Orden Orbiinida $\quad$ Familia Orbiinidae Hartman, 1942 \\
Leitoscoloplos pugettensis Pettibone, 1957 \\
Leitoscoloplos kerguelensis McIntosh, 1885 \\
Scoloplos treadwelli Eisig, 1914 \\
Familia Paraonidae Cerruti, 1909 \\
Aricidea (Aricidea) minima Strelov, 1914 \\
Aricidea minuta Southward, 1956 \\
Aricidea rubra Hartman, 1963 \\
Aricidea simplex Day, 1963 \\
Levinsenia gracilis (Tauber, 1879)
\end{tabular}

Orden Cossurida

Familia Cossuridae Day, 1963

Cossura brunnea Fauchald, 1972

Cossura soyeri Laubier, 1964 
Orden Spionida

Superorden Spioniformia

Familia Spionidae Grube, 1850

Minuspio cirrifera (Wirén, 1883)

Minuspio perkinsi (Maciolek, 1985)

Paraprionospio alata (Moore, 1923)

Paraprionospio pinnata (Ehlers, 1901)

Prionospio cristata Foster, 1971

Prionospio dubia Day, 1964

Prionospio jubata Blake, 1996

Prionospio malmgreni Claparède, 1870

Prionospio vermillionensis Fauchald, 1972

Spiophanes bombyx (Claparède, 1870)

Spiophanes kroeyeri Grube, 1860

Spiophanes wigleyi Pettibone, 1961

Familia Magelonidae Cunningham y Ramage, 1888

Magelona pacifica Monro, 1933

Magelona sacculata Hartman, 1961

Superorden Chaetopteriformia

Familia Chaetopteridae Audouin y Milne-Edwards, 1833 Spiochaetopterus costarum (Claparède, 1869)

Superorden Cirratuliformia

Familia Cirratulidae Ryckholt, 1851

Aphelochaeta parvus Berkeley, 1929

Aphelochaeta monilaris (Hartman, 1960)

Caulleriella gracilis Hartman, 1969

Chaetozone corona Malmgren, 1867

Chaetozone setosa Malmgren, 1867

Monticellina serratiseta (Banse y Hobson, 1968)

Orden Capitellida

Monticellina tesselata (Hartman, 1960)

Familia Capitellidae Grube, 1862

Capitella sp.

Familia Maldanidae Malmgren, 1867

Clymenella complanata Hartman, 1969

Clymenella mucosa (Andrews, 1891)

Clymenura gracilis Hartman, 1969

Maldane sarsi Malmgren, 1865

Praxillella gracilis (Sars, 1861)

Praxillella affinis pacifica Berkeley, 1929

Rhodine bitorquata Malmgren, 1865

Orden Opheliida

Familia Opheliidae Malmgren, 1867

Polyophthalmus pictus (Dujardin, 1839)

Familia Scalibregmatidae Malmgren, 1867

Scalibregma inflatum Rathke, 1843

Orden Phyllodocida

Superorden Aphroditiformia

Superfamilia Aphroditacea

Familia Sigalionidae Kinberg, 1856

Sthenelanella uniformis Moore, 1910

Sigalion spinosus (Hartman, 1939)

Superorden Nereidiformia

Familia Hesionidae Grube, 1850

Hesione intertexta Grube, 1978

Podarkeopsis glabra (Hartman, 1971)

Familia Pilargidae de Saint-Joseph, 1899 Ancistrosyllis jonesi Pettibone, 1966

Hermundura ocularis (Emerson y Fauchald, 1971)

Sigambra bassi (Hartman, 1947)

Sigambra tentaculata (Treadwell, 1941)

Sigambra setosa Fauchald, 1972

Familia Syllidae Grube, 1850

Exogone dispar (Webster, 1879)

Opisthosyllis brunnea Langerhans, 1879

Opisthosyllis japonica Imajima, 1966
Syllis caeca (Katzmann, 1973)

Syllis gracilis Grube, 1840

Trypanosyllis zebra (Grube, 1860)

Trypanosyllis aeolis Langerhans, 1879

Familia Nereididae Blainville, 1818

Ceratocephale papillata De León-Gonzalez y Góngora-Garza, 1992

Neanthes micromma Harper, 1979

Nereis pelagica Linnaeus, 1758

Rullierinereis fauchaldi De León-Gonzalez y Solís-Weiss, 2000

Superorden Glyceriformia

Familia Glyceridae Grube, 1850

Glycera americana Leidy, 1855

Glycera capitata Öersted, 1843

Glycera oxycephala Ehlers, 1887

Glycera tesselata Grube, 1863

Familia Goniadidae Kinberg, 1866

Glycinde polygnatha Hartman, 1950

Goniada brunnea Treadwell, 1906

Goniada maculata Öersted, 1843

Familia Nephtyidae Grube, 1850

Aglaophamus verrilli (McIntosh, 1885)

Bipalponephtys cornuta (Berkeley y Berkeley, 1945)

Nephtys caecoides Hartman, 1938

Nephtys ferruginea Hartman, 1940

Orden Eunicida

Nephtys magellanica Augener, 1912

Superfamilia Eunicea

Familia Eunicidae Berthold, 1827

Eunice americana Hartman, 1944

Eunice vittata (Delle Chiaje, 1828)

Familia Lumbrineridae Schmarda, 1861

Lumbrinereis crassidentata Fauchald, 1970

Lumbrinereis cruzensis Hartman, 1944

Lumbrinereis erecta (Moore, 1904)

Lumbrinereis latreilli Audouin y Milne-Edwards, 1834

Ninoe longibranchiata Fauchald, 1972

Familia Dorvilleidae Chamberlin, 1919

Protodorvillea kefersteini (McIntosh, 1869)

Schistomeringos annulata (Moore, 1906)

Orden Sternapsida

Familia Sternapsidae Carus, 1863

Sternaspis fossor Stimpson, 1854

Orden Flabelligeridae

Familia Flabelligeridae de Saint Joseph, 1894 Pherusa neopapilata Hartman, 1961

Orden Terebellida

Familia Pectinariidae de Quatrefages, 1866

Pectinaria (Amphictene) auricoma (O.F. Müller, 1776)

Pectinaria hartmanae (Reish, 1968)

Pectinaria californiensis Hartman, 1941

Familia Ampharetidae Malmgren, 1866

Amphicteis scaphobranchiata Moore, 1906

Lysippe labiata Malmgren, 1866

Eclysippe trilobata (Hartman, 1969)

Melinna oculata Hartman, 1969

Melinna tentaculata Fauchald, 1972

Melinnampharete gracilis Hartman, 1969

Orden Sabellida

Paralysippe annectens (Moore, 1923)

Familia Sabellidae Latreille, 1825

Paradialychone ecaudata (Moore, 1903)

Euchone incolor Hartman, 1965

Megalomma pigmentum Reish, 1963

Pseudopotamilla reniformis (Müller, 1771)

Familia Serpulidae Rafinesque, 1815

Hydroides gracilis (Bush, 1905) 


\section{Referencias}

Barbosa-López, A. (2008). Análisis latitudinal de los poliquetos de la costa occidental de la península de Baja California. Tesis de maestría. Facultad de Ciencias, Universidad Nacional Autónoma de México.

Cacabelos, E., Moreira, J. y Troncoso, J. (2006). Distribution of Polychaeta in soft-bottoms of a Galician Ria (NW Spain). Scientia Marina, 72, 655-667.

De León-González, J. A. (1988). Mooreonuphis bajacalifornica new sp. a new Onuhid (Polychaeta: Onuphidae) epizoic on the thorny oyster Spondylus princeps unicolor. Revista de Biología Tropical, 36, 443-436.

De León-González, J. A. (1990). Eunice orensanzi, a new eunicid polychaete from the western coast of Baja California Sur, Mexico: with a key to the Mexican species of Eunice. Revista de Biología Tropical, 38, 259-266.

De León-González, J. A. (1992). Soft bottom polychaetes from the western coast of Baja California Sur, Mexico. II. Poecilochaetidae. Cahiers de Biologie Marine, 33, 109-114.

De León-González, J. A. (1994a). Poliquetos (Annelida: Polychaeta) de la plataforma continental de la costa oeste de Baja California Sur, México: taxonomía, hábitos alimenticios y distribución. Tesis de maestría. Centro Interdisciplinario de: Ciencias Marinas. Departamento de Biología Marina. Instituto Politécnico Nacional.

De León-González, J. A. (1994b). Soft bottom polychaetes from the western coast of Baja California Sur, Mexico 4. Onuphidae. Cahiers de Biologie Marine, 35, 57-67.

De León-González, J. A. (1998). Spionidae and Opheliidae (Annelida: Polychaeta) from the western coast of Baja California, Mexico. Bulletin of Marine Science, 60, 7-16.

De León-González, J. A., Hernández-Guevara, N. A. y Rodríguez-Valencia, J. A. (2006). Paraonidae (Polychaeta) from western Mexico, with description of two new species. Journal of the Marine Biological Association of the United Kingdom, 86, 253-262.

De León-González, J. A. y Díaz-Castañeda, V. (1998). Two new species of Nereis (Polychaeta: Nereididae) from Todos Santos Bay, Ensenada, B.C. Mexico. Proceedings of the Biological Society of Washington, 111, 823-828.

De León-González, J. A. y Rodríguez-Valencia, J. A. (1996). Orbiinidae (Polychaeta) from soft bottom of the western coast of Baja California Peninsula, Mexico. Bulletin of Marine Science, 58, 169-174.

De León-González, J. A. y Solís-Weiss, V. (1998). The genus Perinereis (Polychaeta: Nereididae) from Mexican littorals with the redescription of $P$. anderssoni and P. elenacasoae. Proceedings of the Biological Society of Washington, 111, 674-693.

De León-González, J. A. y Solís-Weiss, V. (2000). A review of the Polychaeta family Nereididae from western Mexico. Bulletin of Marine Science, 67, $1-20$.

De León-González, J. A. y Solís-Weiss, V. (2001). Two new species of Nereis (Polychaeta: Nereididae) from the Mexican Pacific. Proceedings of the Biological Society of Washington, 114, 881-886.

Díaz-Castañeda, V. M., de León-González, J. A. y Solana-Arellano, E. (2005). Structure and composition of the polychaete community from Bahía de San Quintín, Pacific coast of Baja California, Mexico. Southern California Academy of Sciences, 104, 75-99.

Díaz-Castañeda, V. M., de León-González, J. A. y Solana-Arellano, E. (2014). Biodiversity of polychaete assemblages in a highly productive lagoon located in Baja California Sur, Mexico. Proceedings of the Biological Society of Washington, 27, 1-14.

Díaz-Castañeda, V. M. y Almeda-Jauregui, C. O. (1999). Early benthic organism colonization on a Caribbean coral reef (Barbados, W.I.): a plate experimental approach. Marine Ecology Progress, 20, 197-220.

Díaz-Castañeda, V. M. y de León-González, J. A. (2007). Comunidades de anélidos poliquetos en Bahía Magdalena, Baja California Sur, México. En R. Funes y J. Gutiérrez (Eds.), Bahía Magdalena: estudios ecológicos (pp. 91-100). La Paz, B.C.S.: Instituto Politécnico Nacional.

Díaz-Castañeda, V. M. y Harris, L. (2004). Biodiversity and structure of the polychaete fauna from soft-bottoms of Bahía Todos Los Santos, Baja California, Mexico. Deep Sea Research Part II: Topical Studies in Oceanography, 51, 827-847.
Díaz-Castañeda, V. M. y San Martín, G. (2001). Syllidae (Polychaeta) from San Quintín Bay, Baja California, Mexico, with a description of a new genus. Proceedings of the Biological Society of Washington, 114, 708-719.

Donath-Hernández, F. E. y Loya-Salinas, D. (1989). Contribución al estudio de la contaminación orgánica marina de la Bahía de Todos Santos, Baja California. Ciencias Marinas, 15, 73-88.

Encalada-Fleites, R. R. y Millán-Núñez, E. (1990). Impacto de las aguas residuales industriales y domésticas sobre las comunidades bentónicas de la Bahía de Todos Santos, Baja California, México. Ciencias Marinas, 16, 121-139.

Fauchald, K. (1968). Onuphidae (Polychaeta) from western Mexico. Allan Hancock Monographs in Marine Biology, 3, 1-82.

Fauchald, K. (1970). Polychaetous annelids of the families Eunicidae, Lumbrineridae, Iphitimidae, Arabellidae, Lysaretidae and Dorvilleidae from western Mexico. Allan Hancock Monographs in Marine Biology, 5, $1-335$.

Fauchald, K. (1972). Benthic polychaetous annelids from deep water of western Mexico and adjacent areas in the Eastern Pacific Ocean. Allan Hancock Monographs in Marine Biology, 7, 1-561.

Fauchald, K. (1982a). Revision of Onuphis, Nothria and Paradiopatra (Polychaeta: Onuphidae) base upon type material. Washington, D.C: Smithsonian Institution, Smithsonian Contribution to Zoology.

Fauchald, K. (1982b). Some species of Onuphis (Polychaeta: Onuphidae) from the Atlantic Ocean. Proceedings of the Biological Society of Washington, 92, 238-250

Gray, J. S. (2000). The measurement of marine species diversity, with an application to the benthic fauna of the Norwegian continental shelf. Journal of Experimental Marine Biology and Ecology, 250, 23-49.

Hartman, O. (1939a). Polychaetous annelids, Part 1: Aphroditidae to Pisionidae. Allan Hancock Monographs in Marine Biology, 7, 1-56.

Hartman, O. (1939b). New species of polychaetous annelids from Southern California. Allan Hancock Pacific Expeditions, 7, 159-171.

Hartman, O. (1940). Polychaetous annelids, 2. Chrysopetalidae to Goniadidae. Allan Hancock Pacific Expeditions, 7, 173-287.

Hartman, O. (1941). Polychaetous annelids, 4. Pectinariidae. Allan Hancock Pacific Expeditions, 7, 325-338.

Hartman, O. (1944a). Polychaetous annelids, 5. Eunicea. Allan Hancock Pacific Expeditions, 10, 1-237.

Hartman, O. (1944b). Polychaetous annelids, 6. Paraonidae, Magelonidae, Longosomatidae, Ctenodrilidae and Sabellidae. Allan Hancock Pacific Expeditions, 10, 239-389.

Hartman, O. (1944c). Polychaetous Annelids, 7. Capitellidae. Allan Hancock Pacific Expeditions, 10, 391-523.

Hartman, O. (1950). Goniadidae, Glyceridae and Nephtydae. Allan Hancock Pacific Expeditions, 15, 1-181.

Hartman, O. (1956). Polychaetous annelids erected by Treadwell, 1891-1948 together with a chronology. Bulletin of American Museum of Natural History, 109, 243-310.

Hartman, O. (1957). Orbiniidae, Apistorbranchidae, Paraonidae and Longosomidae. Allan Hancock Pacific Expeditions, 15, 211-393.

Hartman, O. (1961). Polychaetus annelids from California. Allan Hancock Pacific Expeditions, 1-226.

Heilskov, A. C. y Holmer, M. (2001). Effects of benthic fauna on organic matter mineralization in fish-farm sediments: Importance of size and abundance. Journal of Marine Science, 58, 427-434.

Hernández-Alcántara, P. (2002). Composición y estructura de las comunidades de poliquetos (Annelida: Polychaeta) bénticos de la plataforma continental del Golfo de California. Tesis doctoral. México, D.F: Facultad de Ciencias, Posgrado en: Ciencias Biológicas. UNAM.

Hernández-Guevara, N. A. (1994). Poliquetos (Annelida: Polychaeta) de Bahía Sebastián Vizcaíno, Baja California, México: taxonomía y distribución. Tesis. Facultad de Ciencias Biológicas: Universidad Autónoma de Nuevo León.

Hernández-Valdez, V. D. (2008). Poliquetos bénticos intermareales de la ensenada de La Paz, Baja California Sur, México: ecología comunitaria y detección de especies con potencial acuacultural. Departamento de Posgrado de la Universidad Autónoma de Baja California Sur, La Paz, BCS.

McConnaughey, B. H. (1978). Introduction to marine biology. St Louis: Mosby. 
Rodríguez-Valencia, J. A. (1994). Poliquetos (Annelida: Polychaeta) de la bahía San Juanico, Baja California Sur, México. Tesis. Facultad de Ciencias Biológicas: Universidad Autónoma de Nuevo León.

Ross, D. A. (1977). Introduction to oceanography. Englewood Cliffs, N.J: Prentice-Hall.

Salazar-Vallejo, S. I. (1987). Pilargidae (Annelida: Polychaeta) de México: lista de especies, nuevas especies y biogeografía. Cahiers de Biologie Marine, 27, 193-209.
Salazar-Vallejo, S. I., de León-González, J. A. y Salaices-Polanco, H. (1989). Poliquetos (Annelida: Polychaeta) de México. La Paz: Libros Universitarios, Editorial de la Universidad Autónoma de Baja California Sur:

Schiff, K. C., Allen, M. J., Zeng, E. Y. y Bay, S. M. (2000). Southern California. Marine Pollution Bulletin, 41, 76-93.

Wilson, M. y Shmida, A. (1984). Measuring beta diversity with presenceabsence data. The Journal of Ecology, 72, 1055-1064. 